\title{
Development of Microwave Kinetic Inductance Detectors for phonon and photon detections
}

\author{
H. Ishino*, A. Kibayashi, Y. Kibe, Y. Yamada, A. Okamoto, Y. Kida, \\ Department of Physics, University of Okayama, 3-1-1 Tsushimanaka, Kita-ku, Okayama \\ 700-8530, Japan \\ E-mail: Scishino@s.okayama-u.ac.jp
}

\section{Hazumi, N. Sato}

KEK, High Energy Accelerator Research Organization, 1-1 Oho, Tsukuba, Ibaraki 305-0801, Japan

\section{H. Watanabe}

SOKENDAI, 1-1 Oho, Tsukuba, Ibaraki 305-0801, Japan

\begin{abstract}
We present our recent developments of Microwave Kinetic Inductance Detectors (MKIDs) for phonon and photon detections. Cooper-pair breaking caused by deposited energy of phonons or photons changes the kinetic inductance of a resonator formed with a thin superconducting metal layer. The detection principle of the MKIDs enables us to have a frequency-domain multiplexing. We have developed an array of Lumped Element KID resonators for the detections of phonons from the substrate and visible light photons.
\end{abstract}

Technology and Instrumentation in Particle Physics 2014,

2-6 June, 2014

Amsterdam, the Netherlands

\footnotetext{
* Speaker.
} 


\section{Introduction}

We present our development of Microwave Kinetic Inductance Detectors (MKIDs) for the detections of phonons and photons. The MKIDs consist of resonators and a microwave transmission line (feed line). The resonator has its own resonance frequency, usually tuned from 1 to $10 \mathrm{GHz}$ so as to match the bandwidth of the cryogenic HEMT amplifier. The resonant frequency microwaves are provided from the feed line through the capacitive or inductive coupling between the resonator and the feed line. The MKIDs are made of a single or double thin superconducting metal layer(s). Breaking of Cooper-pairs caused by deposited energy of phonons or photons changes the kinetic inductance of the superconductor and the resonant frequency. This feature provides us the frequency domain multiplexing, enabling us to readout hundreds of resonators with a single wire. Since the dissipation loss of the superconductor for the alternative current is small, the resonant quality factor is usually high, in the order of $10^{4}$ to $10^{6}$, yielding a high sensitivity. The energy needed to break a Cooper-pair is an order of $\mathrm{meV}$, three orders of magnitude lower than the energy to create an electron-hole pair in the semiconductor detector. Therefore, the MKIDs are able to detect electromagnetic waves from millimeter waves to $X$ and $\gamma$ rays as well as the charged particles. Other features of the MKIDs include their simple structures and not requiring detector bias.

The MKIDs were first devised by P. K. Day et al. in 2003 [1], making use of quarter-wave resonators capacitively coupled to a feed line. In 2008, S. Doyle et al. proposed a Lumped Element KID (LEKID) [2] that consists of resonators formed by lumped elements of an inductor and a capacitor; the inductor is a meander line and the capacitor is an interdigital capacitor. The advantage of using LEKID is that the alternative electric current density in the meander structure is almost uniform. Therefore, the sensitivity is independent of the position where energy is deposited, as the sensitivity is proportional to the square of the electric current. On the other hand, the quarter-wave resonator has a higher electric current density at the end of the resonator connected to the ground.

We have developed an array of LEKID resonators for detections of visible photons and athermal phonons from the substrate.

\section{Phonon detection}

We designed an array of LEKID as shown in Fig. 1 The detector consists of 26 LEKID resonators. One LEKID resonator has a size of $0.9 \times 0.8 \mathrm{~mm}^{2}$ with the line width of $12 \mu \mathrm{m}$. The position of the resonators relative to the feed line and neighborhood is optimized so that the resonator has a moderate inductive coupling to the feed line and small cross-talks to other resonators. At the initial stage of the designing, the amount of the cross-talk was evaluated using an electromagnetic simulator, SONNET [3]. We estimated the expected electric current in one resonator when the feed line supplied the resonant frequency microwave to the next resonator. We found the cross-talk becomes smaller as the distance to the next resonator and the difference of the resonator frequencies of adjacent resonators are larger. We tuned the locations and the resonant frequencies of the resonators so as to have the cross-talks less than $1 \%$ and a sensitive area as larger as possible under the constraints of a limited area on the substrate and a limited bandwidth in the readout electronics. 


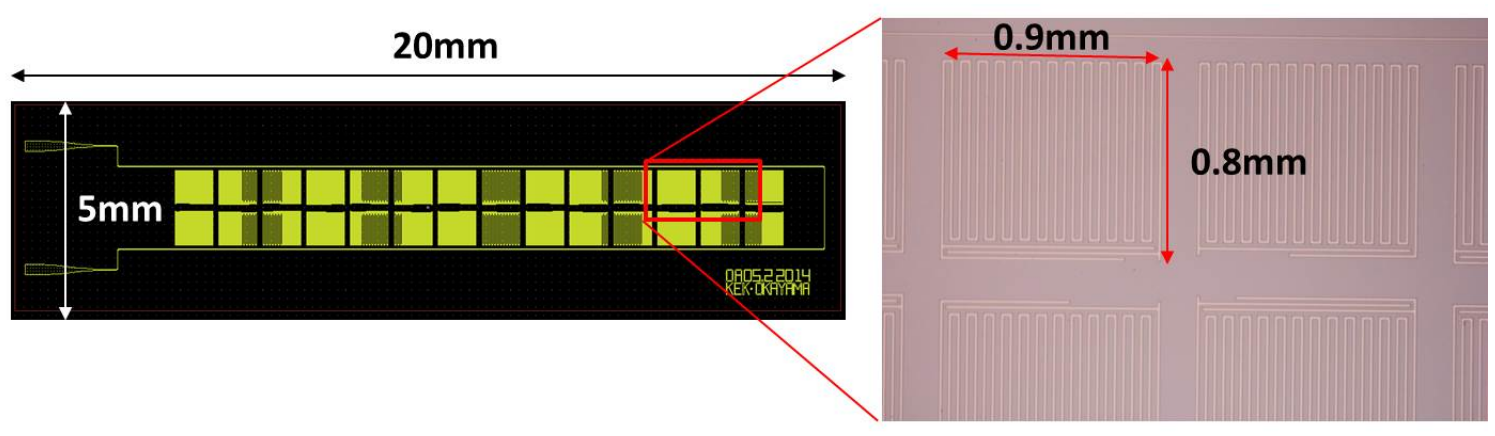

Figure 1: Left: Design of an array of LEKID consisting of 26 resonators. Right: Microscopic picture of the resonators of the fabricated device. The size of each resonator is $0.9 \times 0.8 \mu \mathrm{m}^{2}$ and the width of the line is $12 \mu \mathrm{m}$. Individual resonator has a different resonant frequency tuned by the line overlaps in the interdigital capacitor.

In order to take into account possible differences between the simulation and the real equipment, we fabricated the LEKID device using a $\mathrm{Nb}$ film. As $\mathrm{Nb}$ has the critical temperature of $9.2 \mathrm{~K}$, we are able to quickly iterate the design, fabrication and evaluation of the device using liquid helium and the pumping that create the temperature bath of $1.6 \mathrm{~K}$. We formed the LEKID pattern with the $\mathrm{Nb}$ superconducting metal using a lithography technique at the KEK clean room facility with a lift-off method. We further tuned the design with respect to the feed line coupling to have moderate loading quality factors of the order of $10^{4}$ and the position spacing between the adjacent resonators to have the smaller cross-talk. The cross-talk was measured as the deviation of the resonant frequencies from the designed values. After iterations of the design, fabrication and evaluation, we obtained the final design as shown in Fig. 1.

In order to have a demonstration of the detection of athermal phonons from the substrate, we use the same experimental technique as [4]. We fabricated the detector with a $200 \mathrm{~nm}$ Al layer on a $5 \times 20 \mathrm{~mm}^{2}$ high purity silicon substrate having the thickness of $300 \mu \mathrm{m}$. We use Al to detect the athermal phonons from the substrate, since $\mathrm{Al}$ has the Cooper pair binding energy of $0.34 \mathrm{meV}$, much smaller than that of $\mathrm{Nb}(3.1 \mathrm{meV})$; $\mathrm{Al}$ has a sensitivity to phonons having smaller energy. The device was mounted on a ceramic plate by applying the GE7031 varnish, and covered with a brass housing. We mounted a sealed ${ }^{241} \mathrm{Am}$ source on the housing and irradiated 5.5 $\mathrm{MeV} \alpha$ rays directly to the device. The detector with the radiation source was placed in a ${ }^{3} \mathrm{He}$ refrigerator and cooled down to $0.3 \mathrm{~K}$. Al has the critical temperature of $1.2 \mathrm{~K}$, hence the device is needed to be cooled to $0.3 \mathrm{~K}$ to operate as a detector. We searched for the resonant peaks using a vector network analyzer. For the detector made of a $\mathrm{Nb}$ film, we found the production yield greater than $95 \%$, while the $\mathrm{Al}$ detector had the production yield of about $60 \%$. The reason of the low production yield of the $\mathrm{Al}$ detector is under investigation. Therefore, we were not able to make a unique identification of the resonant peaks to the corresponding locations of the resonators on the substrate.

We made use of the homodyne scheme to readout two resonators' time responses to athermal phonons. Two microwaves, each having the resonant frequency of one of the resonators, were generated using two signal generators. Each microwave was split into two, and one of them was combined with the other microwave and fed into to LEKID in the refrigerator. The output of 

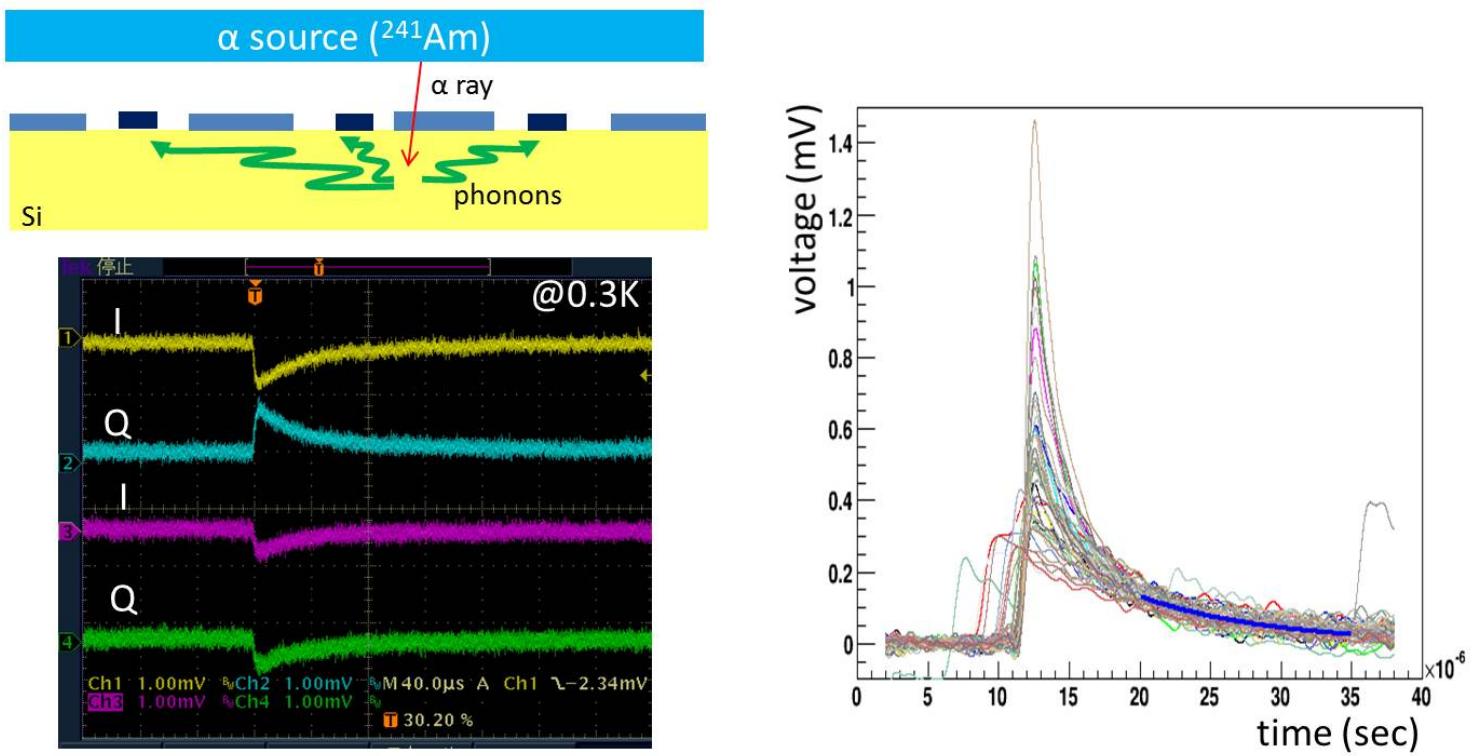

Figure 2: Left: Experimental setup of the $\alpha$ particle irradiation on the device and an oscilloscope image of the detector phonon signal for two resonator's I/Q. Right: Superposition of 50 phonon signals of one resonator. The thick curve in the time range $20 \sim 35 \mu$ s is the fit result of the decay curve.

the LEKID was amplified using a cryogenic HEMT amplifier and a room temperature amplifier, divided into two, and used as the RF input to mixers. The microwave divided at the first step was fed to a hybrid circuit that made two outputs with a relative $\pi / 2$ phase shift, each was the LO (Local Oscillator) input to the mixers. The IF (Intermediate Frequency) outputs of the mixers were the In-phase (I) and Quadrature (Q) signals. Two resonators' I/Q signals were recorded by an oscilloscope with a cutoff frequency of $20 \mathrm{MHz}$, much smaller than the frequency difference of the two microwaves. Figure 2 left shows a schematic view of the $\alpha$ particle irradiation and an oscilloscope image indicating the detection of phonon signals with the coincident timing for two resonators. Figure 2 (right) shows a superposition of 50 phonon signals for one resonator. The maximum pulse height may indicate the location of $\alpha$ particle irradiation: the closer the $\alpha$ particle was irradiated, the higher the maximum pulse height. The decay curves with the time greater than $20 \mu$ s are almost the same height, independent of the maximum height. This may indicate the thermalization of the substrate, that was also observed by [5]. We fit the curve with a time range from 20 to $35 \mu \mathrm{s}$ with a single exponential curve, and obtained $9.3 \pm 0.4 \mu \mathrm{s}$, comparable to the estimated decay time obtained from the thermal capacitance of the substrate and thermal conductance of the varnish.

\section{Photon detection}

Based on the designing technique explained in the previous section, we made a $10 \times 10 \mathrm{~mm}^{2}$ LEKID consisting of 56 resonators, as depicted in the left figure of Fig. 3 We fabricated the detector with a $50 \mathrm{~nm} \mathrm{Nb}$ layer at KEK. The device was inserted to a cryostat filled with liquid 

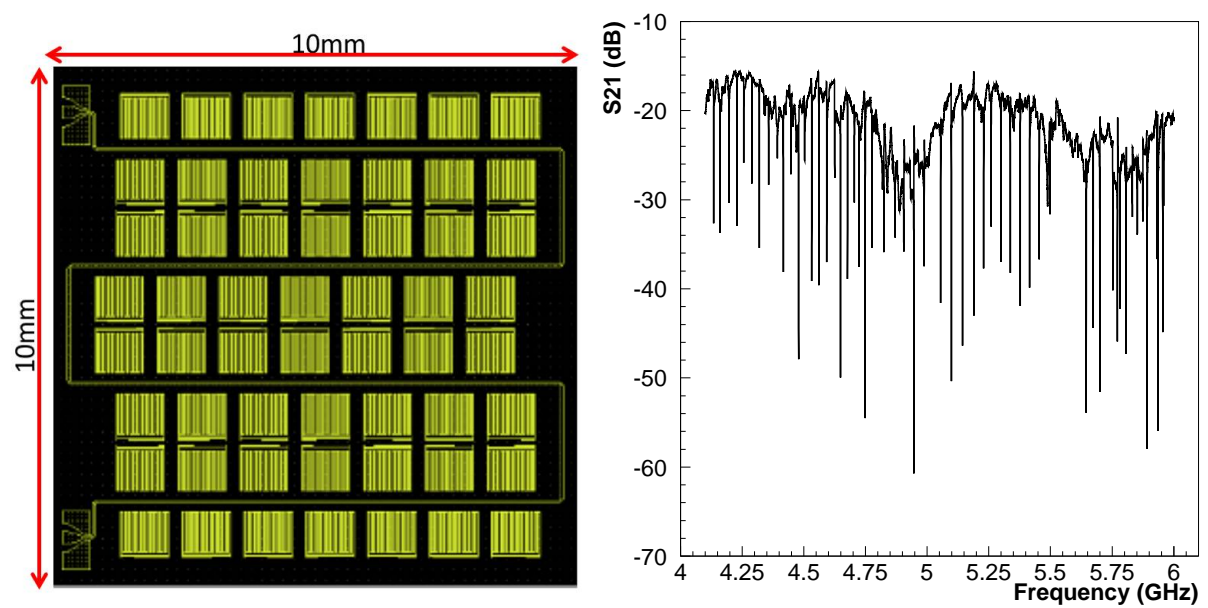

Figure 3: Left: Design of the 56 resonators' LEKID array. The size of each resonator is $0.9 \times 0.8 \mu \mathrm{m}^{2}$. Right: Resonant peaks measured by a vector network analyzer. The horizontal (vertical) axis shows the frequency in $\mathrm{GHz}\left(S_{21}\right.$ in $\left.\mathrm{dB}\right)$.

helium that was pumped and cooled to $1.6 \mathrm{~K}$. Figure 3 (right) shows the resonant peaks measured using a vector network analyzer. We found 55 resonant peaks, corresponding to a $98 \%$ production yield, in the frequency range of $4 \sim 6 \mathrm{GHz}$ with a $30 \mathrm{MHz}$ spacing, consistent with the expectation of the designed values.

We irradiated visible photons having the wavelength of $660 \mathrm{~nm}$ where used to irradiate the device through an optical system consisting of a low temperature compatible optical prism and an optical fiber, as shown in Fig. 4 We injected photons from a laser (OBIS660) located outside of the cryostat into the optical system immersed in the pumped liquid helium. The prism bended the light from the optical fiber by 90 degrees and the light beam was illuminated onto the device. The photons break Cooper-pairs in the superconductor and change the surface impedance of the resonators, and hence the resonant frequencies. Fig. 4 demonstrates the change of the resonant frequency measured using a vector network analyzer with and without the light injection. We measured a ratio of $\delta f / f_{0}$ for individual resonators, where $\delta f$ is the frequency change and $f_{0}$ is the resonant frequency without the light. Figure 4 shows the illuminated light beam profile; the color corresponds to the magnitude of the ratio $\delta f / f_{0}$. We roughly measured the light beam spot size of $8 \mathrm{~mm}$ at FWHM in our optical system.

We also injected a light pulse with a $10 \mathrm{~ns}$ width. The pulsed laser signal was generated using a system consisting of a laser (OBIS660) and a pulse generator generating $10 \mathrm{nsec}$ TTL pulses that modulated the output signal of the laser. The light yield of the pulsed signal was measured using a photomultiplier (PMT) as a function of the output power of the laser, taking into account the quantum efficiency of the PMT. We chose a resonator located at the center of the beam profile, and recorded the I/Q signals using an oscilloscope. We varied the laser output power and reduced it until we could see the pulse signals of the resonator. By assuming the profile shape is a Gaussian with FWHM $(8 \mathrm{~mm})$, and by using the values of the reflectivity of $\mathrm{Nb}$ at $660 \mathrm{~nm}$ wavelength of $50 \%$, and the fraction of sensitive area of the resonator, we estimated the minimum number of photons detected to be 9 , corresponding to $17 \mathrm{eV}$, as a very preliminary result. 


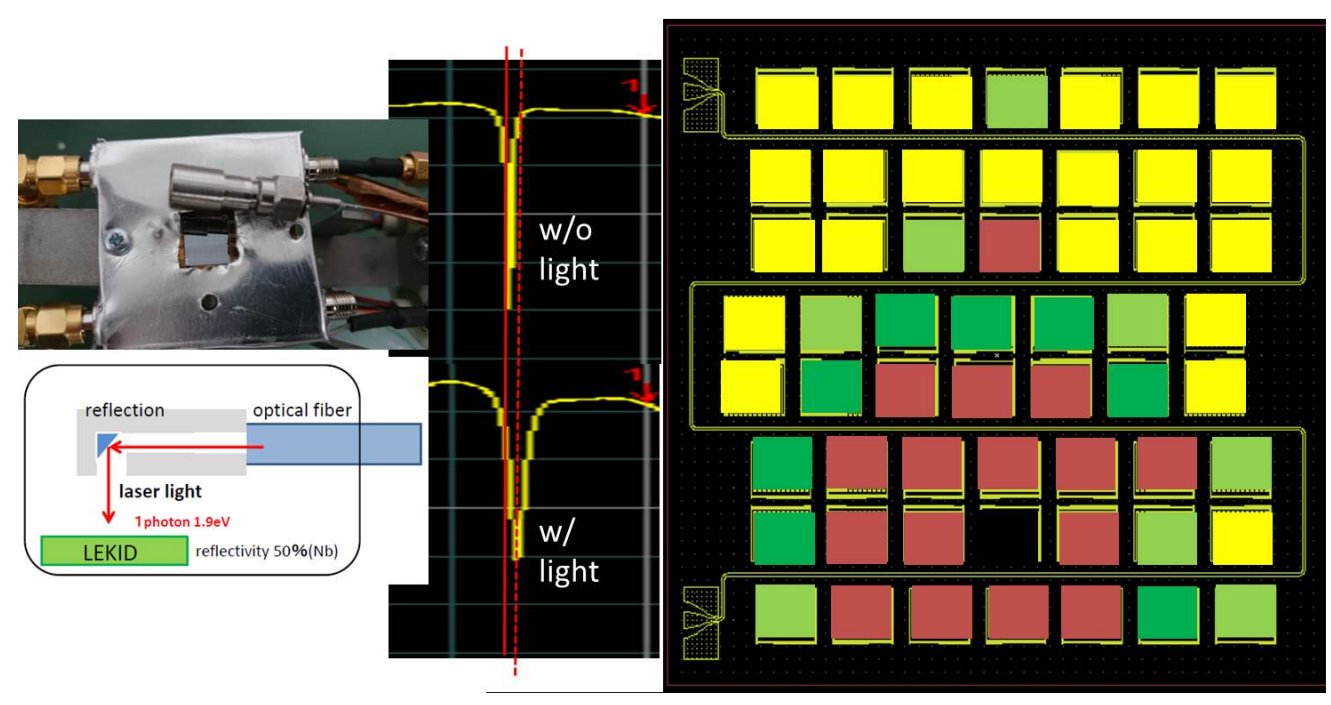

Figure 4: Left: Optical system to irradiate photons to the device. Middle: Resonant frequency change with and without the light. Right: Obtained image of the light beam profile. The individual color corresponds to the magnitude of $\delta f / f_{0}$.

\section{Possible applications}

We aim to use the superconducting detectors we developed to two applications.

First, we are trying to develop a time projection chamber (TPC) using liquid helium for the purpose of a search for light dark matter (Fig. 5]). This detection concept was first proposed by [7]. The recoil helium produces scintillation UV photons of the average energy of $16 \mathrm{eV}$. The ratio of the number of initial scintillation photons (S1) to the ionized electrons (S2) allows us to discriminate the recoils of a helium nucleus and an electron. Since the scintillation light has a short wavelength, a combination of PMTs and wavelength shifters is usually applied to the scintillation light pulses, which reduces the detection efficiency and the discrimination power. On the other hand, the absorption probability of $\mathrm{Nb}$ for a $16 \mathrm{eV}$ photon is about $80 \%$ [6]. A LEKID array we have been developing may be able to detect such photons directly in liquid helium with a higher detection efficiency.

The other application, we propose here for the first time, is a device combining the LEKID with Silicon On Insulator (SOI) as a high resolution X-ray detector. The energy deposited by X-ray photons in the substrate can be measured using superconducting detectors formed at the surface of the substrate through Cooper pair breaking caused by the athermal phonons. Since the binding energy of Cooper pair is in the order of $\mathrm{meV}$, three orders of magnitude lower than that of the semiconductor detectors, superconducting detectors in principle have the energy resolution much higher than that of semiconductor detectors. In fact, by using superconducting tunnel junctions (STJs) Kurakado et al. [8] obtained 1.2\% energy resolution for $5.9 \mathrm{keV} \mathrm{X-ray} \mathrm{photons,} \mathrm{about} \mathrm{a} \mathrm{half}$ of the principle energy resolution (2\%) of the semiconductor detectors. The resolution was limited by the position correction ability; the difficulty of reading out STJ signals with high multiplicity limited the number of readout channels of four, degrading the position resolution of about $1 \mathrm{~mm}$. 

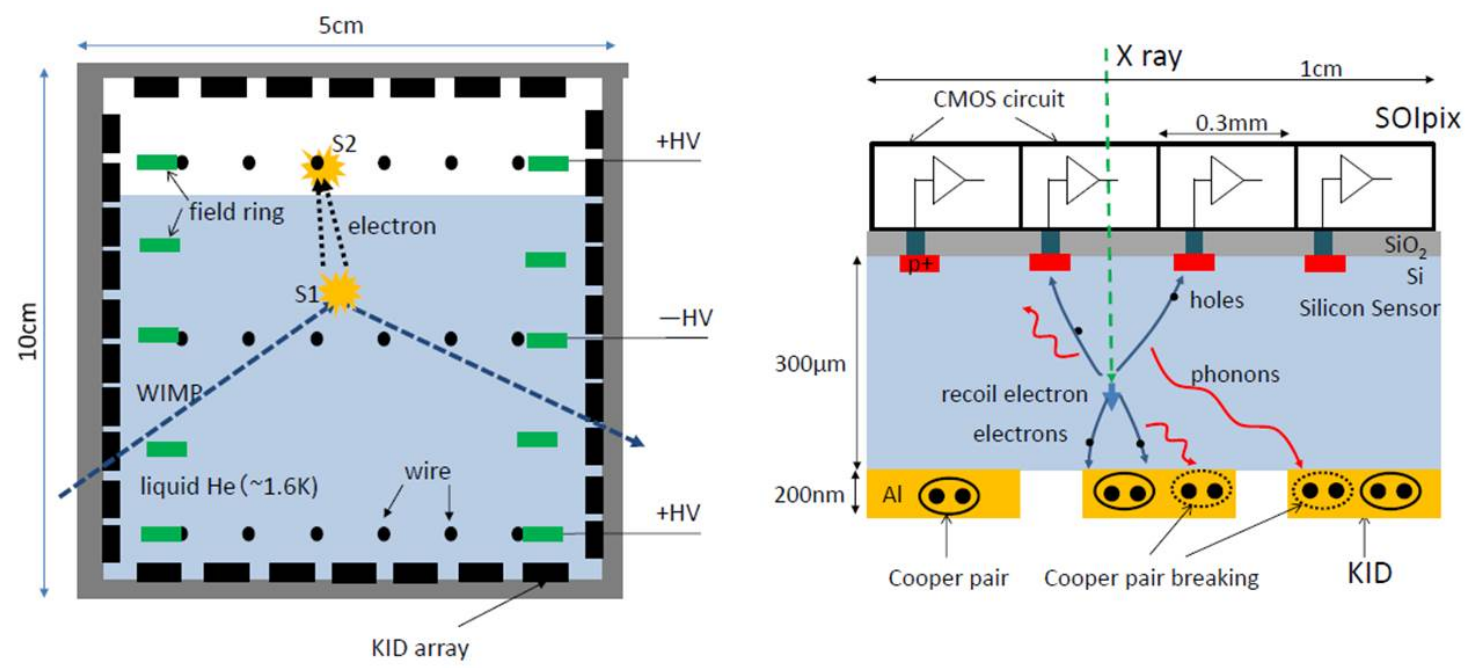

Figure 5: Left: He-TPC with LEKIDs for the direct detection of scintillation photons. Right: Newly proposed device combining SOI pixel detector and LEKID.

The same detection method employing LEKID resonators was conducted by Moore et al. [5], who obtained the energy resolution of $550 \mathrm{eV}$ at $30 \mathrm{keV}$ after the position correction with the resolution of $\leq 1 \mathrm{~mm}$. The higher position resolution may improve the energy resolution and be achieved using a SOI pixel sensor able to function temperatures lower than $1 \mathrm{~K}$. We propose the combination of SOI pixel sensor [9] and LEKID as shown in Fig. 5 The SOI pixel detector senses the ionization signal and identifies the position with a $0.1 \mathrm{~mm}$ precision, while LEKID processed on the backside of the substrate detects athermal phonons to measure the total energy. The key issue, however, is to develop a low power consumption SOI pixel detector. We started a dedicated R\&D to tackle this problem.

\section{Summary}

We have developed the LEKID for the detection of phonons and photons. The LEKID made of $\mathrm{Al}$ detected athermal phonons produced by $\alpha$ particles in the Si substrate. The decay time of the phonon signal is comparable to the estimation of the substrate thermal capacitance and thermal conductance to the ceramic plate. We have developed an array of 56 resonators of LEKID fabricated using $\mathrm{Nb}$. The $660 \mathrm{~nm}$ visible photons were detected successfully; we took an image of the light beam profile and detected $10 \mathrm{nsec}$ pulse signals down to 9 photons. We aim to apply the LEKID developed so far to a liquid helium TPC and a device combined with SOI pixel detector.

\section{Acknowledgments}

The authors would like to thank KEK Detector Technology Project for the support of the development of superconducting detectors at KEK. This work is supported by the Grand-in-Aid for Scientific Research (B) No. 26287047. 


\section{References}

[1] P. K. Day et al., Nature 425 (2003) pp.817-820.

[2] S. Doyle et al., J. Low Temp. Phys. 151 (2008) pp.530-536.

[3] https://www.sonnetsoftware.com/.

[4] H. Ishino et al., J. Low Temp. Phys. 176, (2014) pp.161-167.

[5] D. C. Moore et al., Appl. Phys. Lett. 100, 232601 (2012) pp.1-4.

[6] J. H. Weaver, D W. Lynch and C. G. Olson, Phys. Rev. B 7, (1973) pp.4311-4318; T. Peacock et al., Astron. Astrophy. Suppl. 123, (1997) pp.581-587.

[7] W. Guo and D. N. McKinsey, Phys. Rev. D 87, 115001 (2013) pp.1-15.

[8] M. Kurakado et al., Nucl. Instr. Meth A 621, (2010) pp.431-436.

[9] Y. Arai et al., Nucl. Instr. Meth A 636, (2011) pp.S31-S36. 